\title{
EFFECT OF ACCUMULATION LAYER ON TUNNELING IN ZnSe-ZnTe DOUBLE BARRIER HETEROSTRUCTURES*
}

\author{
P. Bala, R. Ryszewski, W. Bala \\ Institute of Physics, N. Copernicus University, Grudziądzka 5, 87-100 Toruń, Poland
}

The tunneling probability in double barrier heterostructures can be affected by various effects. One of the most significant is presence of the accumulation layer placed before the structure. The presented time-dependent results show that charge trapped in the accumulation region oscillates in the triangular quantum well and tunnels sequentially through the double barrier structure resulting in periodical changes of the charge density right to the heterostructure.

PACS numbers: 03.65.-w, 73.40.Gk

Tunneling phenomena in superlattices take big attention both in theoretical and experimental aspects because of fundamental interest as well as possible applications. Because of complicated descriptions of tunneling most of the theoretical works is limited to the ideal heterostructures with sharp step-like potential edges. This assumptions may not be satisfied in real semiconductor devices according to the technology requirements. The additional effect can be caused dynamically by the changes in the electron density near the structure which changes band structure of the system [1]. Another source of modification of the electronic structure can be introduced, but presence of charge accumulation layer in the region near energy barrier is the most common effect which can affect operations of heterostructures devices [2].

In this paper the tunneling phenomena in $\mathrm{ZnSe}-\mathrm{ZnTe}$ double barrier heterostructure are investigated using time-dependent quantum mechanics of wave packet as well as classical planar wave approach. As it has been shown previously those methods allow accurate and detailed description of the tunneling phenomena for various heterostructures $[3,4]$.

The double barrier resonant tunneling structure is formed by $n$-type $\mathrm{ZnSe}$ layer of width $d_{\mathrm{W}}=47.6 \AA$ (quantum well) surrounded by $p$-type $\mathrm{ZnTe}$ layers of width $d_{\mathrm{B}}=26.4 \AA$ (barrier region). The height of the barrier is $V_{0}=0.59 \mathrm{eV}$.

*This work is supported in part by the State Committee for Scientific Research (Republic of Poland) under grant No. 2 P302 01905. 
The double barricr structure is placed between wide $n$-type $\mathrm{ZnSe}$ layers (with length greater than $1000 \AA$ ). The system is investigated under constant voltage $V_{z}$ applied along the growth direction. Because of the high resistivity of the $\mathrm{ZnTe}$ layers potential changes only in the barrier regions are assumed. The accumulation layer is modeled by the triangle quantum well of the width $d_{\mathrm{A}}$ and energy depth $V_{\mathrm{A}}$ located left to the heterostructure. The parameters of the well are selected to be independent on applied voltage and accumulated charge whith allows us to investigate direct effect of accumulation layer. The energy diagram of the structure is presented in the inset in Fig. 1.

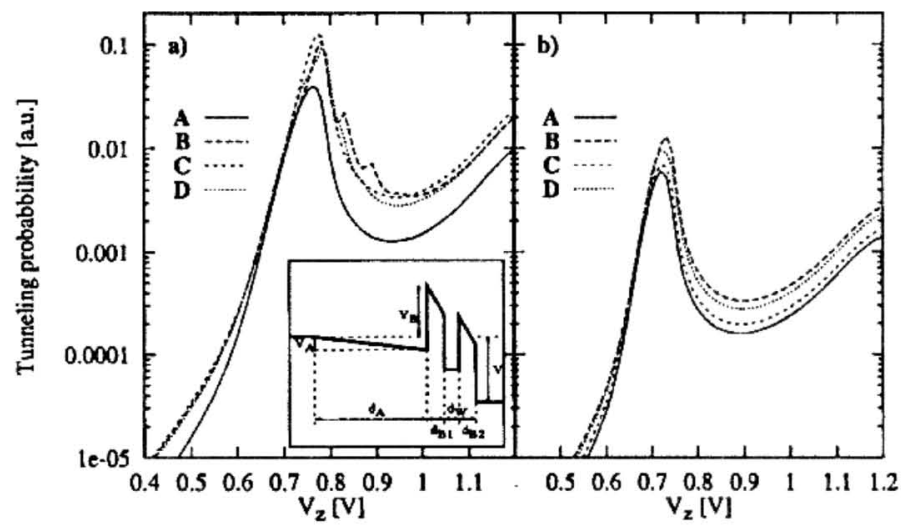

Fig. 1. I-V characteristics of the double barricr $\mathrm{ZnTe} / \mathrm{ZnSe} / \mathrm{ZnTe}$ structure with different accumulation layer parameters obtained within time-dependent (a) and stationary (b) model. The inset presents energy diagram for the double barrier structure with the applied voltage $V$.

In the time-dependent method (TD) the detailed electron dynamics is obtained by solving, within effective mass approximation [5], the time-dependent Schrödinger equation

$$
\mathrm{i} h \frac{\partial \Psi(x, t)}{\partial t}=\left(-\frac{\hbar^{2}}{2 m^{*}} \frac{\partial^{2}}{\partial x^{2}}+V(x)\right) \Psi(x, t),
$$

where $m^{*}$ is the electron effective mass and depends on the layer type ( $m_{\mathrm{ZnSe}}^{*}=$ $0.16 m_{\mathrm{e}}, m_{\mathrm{ZnTe}}^{*}=0.32 m_{\mathrm{e}}$ ). The time-dependent Schrödinger equation (1) can be solved using effective and powerful numerical methods as it was reported previously [3] and in a particular case an algorithm based on the Chebychev polynomial expansion [6] has been used. The initial wave function is of the Gaussian shape, and its parameters, time step and the grid size are the same as in the previous papers [4].

Within the stationary approach (SA) the wave function is obtained by solving the time-independent Schrödinger equation

$$
\frac{\partial^{2} \Psi(x)}{\partial x^{2}}+\frac{2 m^{*}}{\hbar^{2}}[E-V(x)] \Psi(x)=0 .
$$


The tunneling probability can be calculated assuming plane wave as the asymptotic shape of the wave function in the region outside the double barrier heterostructure and the linear combination of the Bessel functions inside the triangular well [4].

The time-dependent and stationary tunneling probability was calculated for different parameters of the accumulation layer and compared to one obtained for the unmodified structure (referred as structure $A$ ). The width of the accumulation layer $d_{\mathrm{A}}=79.4 \AA$ (case $B$ and $C$ ), $132.3 \AA$ (case $D$ ) is larger than width of the initial electron wave function and depth $V_{\mathrm{A}}=0.136 \mathrm{eV}(B$ and $D), 0.272 \mathrm{eV}(C)$ is smaller than the barrier height.

The tunneling probability calculated with both time-dependent and stationary approach exhibits a single resonant peak at the voltage $V_{\mathrm{p}}=0.78 \mathrm{~V}$. As is presented in Fig. 1a, with presence of the accumulation layer small additional maxima at higher voltage are observed on the time-dependent tunneling probability characteristics. With the increase in the width and depth of the accumulation layer the amplitude of the additional peaks decreases. This process is associated with the small increase in the amplitude of the main resonant peak shifted slightly (by $0.02 \mathrm{~V}$ ) to the higher voltage compared to the position with absence of the accumulation region. The additional maxima are equally spaced and can be clearly interpreted as the result of presence of the accumulation layer. This feature, however, is not observed in the tunneling probability calculated with the stationary method (Fig. 1b). The very small contribution to the tunneling probability is observed due to presence of the discrete energy levels originating from triangular well, but hidden in the continuum of the energy accessible in the region left to the structure.

The presented results can be explained by the presence of the quantized energy levels in the triangular quantum well directly before double barrier heterostructure. The incoming wave packet scatters from the first barrier and starts to occupy discrete levels in the accumulation layer region and the resonant tunneling conditions are satisfied not only for the initial energy of the wave function (main resonant peak) but also for the energy levels in the accumulation layer. The energy of these levels is lower than initial energy and additional peaks for higher applied voltages are obtained. Because of absence of the dissipation processes the occupancy of the levels in the triangular quantum well is low which indicates observed small amplitude of the additional peaks. In the stationary approach the wave function is described as planar wave of the accessible energy and the levels in the triangular quantum well do not contribute to the tunneling probability indicating absence of the additional peaks.

The total probability of finding electron in the different spatial regions versus time with $(B)$ and without $(A)$ accumulation layer is presented in Fig. 2a. As it can be detected from the time-dependent tunneling probability the total probability of finding electron right to the barrier is higher with presence of the accumulation layer and starts to increase $0.02 \mathrm{ps}$ faster than without accumulation layer. Paradoxically due to the effective tunneling the probability of finding electron in the accumulation layer is smaller than in the same region but without triangular quantum well and the scattering process is faster. Slightly smaller slope of the 


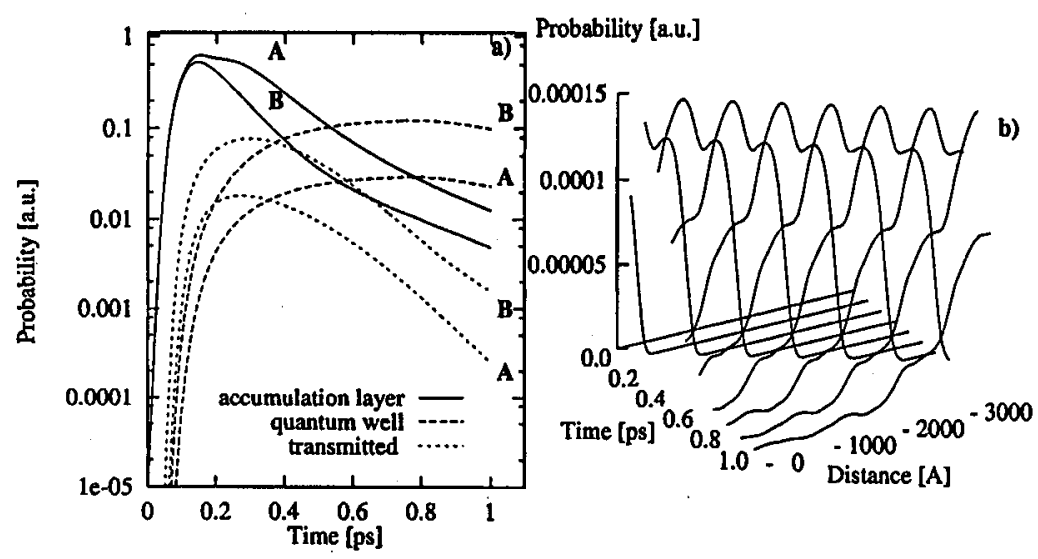

Fig. 2. Time dependence of the total probability of the finding electron in the different spatial regions: (a) for the case $A$ and $B$ and the transmitted wave function, (b) for $d_{\mathrm{A}}=79.4 \AA$ and $V_{\mathrm{A}}=0.136 \mathrm{eV}$.

decay of the total probability in the accumulation layer indicates trapping of the outgoing wave function in the triangular well.

In a result the wave function transmitted through the double barrier resonant structure can be decomposed to the few overlapping wave packets separated in time by $0.2 \mathrm{ps}$. As it is shown in Fig. $2 \mathrm{~b}$ for the case $B$ the highest probability is associated with the second wave packet transmitted. This depends on the parameters of the accumulation layer and highest value of the first probability maximum is obtained for the higher depth of the accumulation layer (case $D$ ). The sequential tunneling is simple consequence of the periodic oscillations of the wave function in the triangular quantum well left to the double barrier structure.

\section{References}

[1] Y. Fu, M. Willander, Phys. Rev. B 44, 13631 (1991).

[2] M. Wagner, H. Mizuta, Appl. Phys. Lett. 63, 2268 (1993).

[3] P. Bata, J.S. Kwiatkowski, W. Bała, Acta Phys. Pol. A 82, 649 (1992).

[4] P. Bała, R. Ryszewski, W. Bała, Acta Phys. Pol. A 84, 685 (1993).

[5] R. Tsu, L. Esaki, Appl. Phys. Lett. 2, 562 (1973).

[6] R. Kosloff, H. Tal-Ezer, Chem. Phys. Lett. 127, 223 (1981). 